\title{
ML-SA1, a TRPML1 agonist, induces gastric secretion and gastrointestinal tract inflammation in vivo
}

\author{
Dan GONG ${ }^{\#}$, Jing HAl${ }^{\#}$, Jun MA, Chen-Xi WANG, Xin-Dan ZHANG, Ya-Nan XIANG, Tao TAN, \\ Ya-Nan LIU, Wei Kevin ZHANG* \\ Institute of Pharmacology, College of Pharmaceutical Sciences, South-Central University for Nationalities, Wuhan, China \\ \# These authors contributed equally to this work. \\ *Correspondence: wkzhang81@139.com \\ https://doi.org/10.37175/stemedicine.v1i1.3
}

\begin{abstract}
Background: The effect of ML-SA1, a potent and specific TRPML1 channel agonist, on gastric secretion and subsequent impact to the gastrointestinal $(\mathrm{GI})$ tract of mice was investigated.

Methods: Twenty mice were divided into two groups, and were treated with ML-SA1 and dimethyl sulfoxide (as negative control), respectively. The intestinal propulsion rate of the mice was monitored. Stomach and intestinal tissues were sectioned for periodic acid Schiff (PAS), histopathological and immunohistochemical (IHC) analysis. TRPML1 expression level in AGS cells was assayed via Western blot, and calcium imaging was performed in AGS cells upon performing GCaMP5G transfection.

Results: Application of $150 \mu \mathrm{g} / \mathrm{kg} \mathrm{ML-SA} 1$ could result in significant decrease in intestinal propulsion rate and structural changes of the parietal cells in stomach and goblet cells in intestine of mice. Moreover, it induced inflammation to the duodenum section of intestine in mice. IHC staining also revealed that ML-SA1 could induce up-regulation of TRPML1 expression in both the parietal cells of stomach pits and the columnar epithelial cells of duodenum villi. Further analysis of AGS cells, a type of stomach cell line, demonstrated that ML-SA1 could enhance the expression of TRPML1 and induce the protrusion of micro-vesicles.

Conclusion: Our results suggested that ML-SA1 was capable of activating TRPML1 in stomach cells and therefore, leading to increased secretion of gastric acid. This provided a convincing attempt of applying ML-SA1 in animals and pointed out a new possible research direction, that TRPML1 channel could be a potential new therapeutic target on hypochlorhydria or even in the field of gastrointestinal cancer research.
\end{abstract}

Keywords: Mucolipin-1 · Hypochlordria · Gastric cells · Goblet cells · Calcium · COX-2

\section{Introduction}

Gastric acid, which is secreted by the stomach, is mainly composed of hydrochloric acid and divalent ions including potassium and sodium. Current understanding has shown that it is primarily produced by the parietal cells that line the stomach, and plays an initiative role in food digestion and pathogen protection in the gastrointestinal (GI) tract (1). Hence the lack of gastric acid production, or sometimes referred to as hypochlorhydria (achlorhydria or low stomach acid as well), would lead to malnutrition. In addition, hypochlorhydria has been implicated to be

(c) The Author(s). 2019 This is an Open Access article distributed under the terms of the Creative Commons License (http://creativecommons.org/licenses/by/4.0/) which permits unrestricted use, distribution, and reproduction in any medium or format, provided the original work is properly cited. correlated with various disorders in the GI tract including pernicious anemia, Helicobacter pylori (H. pylori) infection, vipomas, hypothyroidism, and gastric cancer. The deficiency is also present in patients who have antisecretory medications, gastric bypass, and radiation to the stomach (1-3). Currently, there is no specific treatment that targets hypochlorhydria. Instead, multiple case-dependent treatments have been used to reduce the complications of hypochlorhydria. For instance, $H$. pylori eradication has been instructed to patients after the infection. Increased uptake of calcium, iron, and/or vitamin D have also been suggested to hypochlorhydria patients (4).

A recent research has demonstrated that TRPML1 (also named mucolipin-1), a protein that is mutated in type-VI mucolipidosis (ML-IV), could be essential for 
tubulovesicles (TV) exocytosis and acid secretion in parietal cells (5). TRPML1 is a non-selective cation channel primarily localized in the late endo-lysosome compartment (6). It belongs to the TRP channel superfamily and is an inwardly rectified channel capable of transporting ordinary cations such as $\mathrm{Na}^{+}, \mathrm{K}^{+}$and $\mathrm{Ca}^{2+}$ (7), as well as some heavy metal ions such as $\mathrm{Fe}^{2+}$ and $\mathrm{Zn}^{2+}(8)$, from the extracellular space or the lumen of the lysosome to the cytosol (9). Many investigations have suggested that the abnormality (loss or down-regulation) of TRPML1 function could result in an accumulation of heterogeneous materials within lysosomes (10) and affect the regulation of lysosome motility, positioning and tubulation (11). Such changes in turn are hypothesized to affect many intracellular processes including membrane trafficking, phagocytosis and vesicular fusion and fission (12).

In this study, we aimed to investigate whether ML-SA1, a specific TRPML1 agonist (13), could induce acid production and secretion to the stomach in a mouse model. Moreover, we also wanted to evaluate the potency of ML-SA1 and the direct consequence of its application in the GI tract in vivo.

\section{Materials and Methods \\ Reagents}

Dimethyl sulfoxide (DMSO, \#D5879) of analytical reagent grade was obtained from Sigma-Aldrich (St Louis, MO, USA). ML-SA1 was provided by Abcam (\#ab144622, UK).

\section{Animal care}

The experimental protocols and animal care and utilization were implemented on the basis of the Guide for Nationalities and the Committee of Research Facilities for Laboratory Animal Sciences, South-Central University for Nationalities, China. The Committee on the Ethics of Animal Experiments of the South-Central University for Nationalities, China (permit number: 2018-SCUECAEC-011) developed related protocols. A great effort in minimizing suffering contained anesthesia by $\mathrm{CO} 2$ before sacrifice.

\section{Cell culture and transfection}

AGS cells (\#CRL-1739, ATCC) were purchased and maintained as described by the instructions. F-12K medium (30-2004, ATCC) was used for maintenance of normal culture, with fetal bovine serum (FBS, Gibco, Thermo Fisher Scientific) being added to a final concentration of $10 \%$. Hieff Trans liposomal transfection reagent (\#40802ES02, Yeasen Inc. China) was used for transfection. pCMV-GCaMP5G plasmid (\#31788) was obtained from Addgene.

\section{Intestinal propulsion rate testing}

Prior to experiment, twenty female Kunming mice (8 weeks old, 20-22 g) were acclimatized for 4 days in pathogen-free conditions. Experimental animals were maintained with basal diet. They were also kept in temperature-controlled animal room $\left(22-25^{\circ} \mathrm{C}\right)$ with at least 4 rounds of $12 \mathrm{~h}$ light-dark cycle before experiments. During acute experiments, the animals were divided into two groups of 10 mice each at random. The treatment group received $150 \mu \mathrm{g} / \mathrm{kg}$ ML-SA1 while the control group received $150 \mu \mathrm{g} / \mathrm{kg}$ DMSO. Both groups received their respective drug every $12 \mathrm{~h}$ for 3 times by gavage. Mice were fasted overnight, and then received an oral administration of $200 \mu \mathrm{l} /$ animal carbonic ink. Fifteen min later, animals were euthanized by cervical dislocation. The stomach and the complete intestinal tract from the pylorus to the terminal rectum were removed. The length of the whole intestinal tract and the distance of ink propulsion were measured. The percentage of blackened intestinal tracts was calculated using the following equation: intestinal propulsion rate $(\%)=$ pushing length/ total length $\times 100 \%$.

\section{Histopathological analysis of tissues}

Fresh stomach and intestines tissue samples were fixed in $4 \%$ polyformaldehyde, embedded in paraffin, cut into $2 \mu \mathrm{m}$ slices. Standard techniques were used for staining with hematoxylin and eosin (H\&E) or hematoxylin alone. Histopathologic examination of the sections was conducted using a Nikon 50i light microscope (Nikon Inc.).

\section{Immunohistochemical (IHC) analysis of tissues}

The tissue sections were deparaffinized and hydrated. The sections were then immersed in $0.01 \mathrm{M}$ boiling citrate buffer and were heated using a microwave oven for 5 min for antigen retrieval. Incubation with 3\% hydrogen peroxide in methanol for 10 min was performed to block endogenous peroxidase activity. After blocking with 5\% serum (AR1009, AR1010; Boster, China) for $1 \mathrm{~h}$, the sections were incubated overnight at $4{ }^{\circ} \mathrm{C}$ with primary antibodies against TRPML1 (1:200, ab28508; Abcam, UK) or COX-2 (1:100, ab23672; Abcam, UK). After incubation with $\mathrm{IgG}$ secondary antibodies (414341C, $414351 \mathrm{C}$, Nichirei, Japan) at $37^{\circ} \mathrm{C}$ for $1 \mathrm{~h}$, the presence of TRPML1 was detected using the DAB detection kit (425314F, Nichirei, Japan). Sections were counterstained with hematoxylin and coverslips were used for mounting.

\section{Periodic Acid Schiff (PAS) Staining}

The samples were sectioned and placed on slides, which were then deparaffinized, rehydrated, oxidized ( $1 \%$ periodic acid for $15 \mathrm{~min}$ ), washed by distilled water and stained with Schiff's reagent for $30 \mathrm{~min}$. Then the sections were washed with hydrochloric acid and $\mathrm{H}_{2} \mathrm{O}$ for 2 min and three times, followed by immersing in tap water for 5 min. Finally, the sections were counterstained with hematoxylin for 20 seconds and dehydrated.

\section{Western blotting}

After treatment, cells were lysed with RIPA protein lysis buffer (Beyotime, China) containing phosphatase inhibitor 
cocktail (100x) (Cwbio, China), protease inhibitor cocktail (100x) (Cwbio, China) and phenylmethylsulfonyl fluorid (Beyotime, China). The concentration of total protein was detected by the Lowry method. Equal amounts of protein were fractionated by $12.5 \%$ sodium dodecyl sulfate polyacrylamide gels and transferred to polyvinylidene difluoride membranes (Millipore Corporation, USA). After blocked with 5\% nonfat dry milk, the membranes were incubated with anti-TRPML1 antibody at $4^{\circ} \mathrm{C}$ overnight. To remove unbound primary antibodies, the membranes were washed 3 times for 5 mins by TBST (Tris-Buffered Saline, 0.1\% Tween-20) solution and followed by exposure to secondary antibodies (1:3000, goat anti-rabbit, Abbkine, USA) for $1 \mathrm{~h}$ at room temperature. Finally, after wash, bands on the membranes were visualized by developer and fixing solution. The protein bands were quantified using the ImageJ software.

\section{Fluorescent microscopy}

Fluorescent signals from GCaMP5G protein were excited with a FITC filter (Ex 465-495, Dm 505, RA 512-558, Nikon) and were collected using an inverted microscope (Eclipse Ti, Nikon) with a color camera (DS-Ri2, Nikon). ImageJ (NIH) software was used to quantify and analyze the obtained signal.

\section{Statistical analysis}

All results were presented as mean \pm SEM. Prism 7 were used for the storage and analysis of data. Student's t-test was performed to compare the difference of groups and the $\mathrm{p}$ value was indicated in the graph. Statistical differences of $p<0.05$ were considered to be significant.

\section{Results}

\section{Application of ML-SA1 reduced intestinal propulsion} rate in mice.

Gavage application of TRPML1 agonist, ML-SA1 (Figure 1A, $150 \mu \mathrm{g} / \mathrm{kg}$, thrice prior to the intestinal propulsion rate test) resulted in a significant decrease in the propulsion rate in mice (Figure 1C and D), from $55.0 \pm 9.0 \mathrm{~cm}$ in vehicle-treated group to $41.6 \pm 7.5 \mathrm{~cm}$ in ML-SA1-treated group. This hold-back effect of ML-SA1 could normally even be greater, taking into consideration that there was only a 15-min gap between the gavage application and sacrifice of mice.

\section{Application of ML-SA1 altered the structure in stomach and duodenum of mice.}

After the above experiment, we sacrificed the animal and took the stomach and duodenum out to further characterize the changes caused by ML-SA1 to the mice. H\&E staining revealed that application of $150 \mu \mathrm{g} / \mathrm{kg}$ ML-SA1 altered the structure of stomach pits and duodenum villi. As shown in Figure $\mathbf{2 A}$, the integrity and order of the pits remained in the stomach of mice. However, more parietal cells were observed in the upper edge of pits in stomach of ML-SA1 treated mice. More importantly, the shape of parietal cells became smaller and more vacant in ML-SA1-treated mice comparing with that of vehicletreated ones, suggesting that ML-SA1 might stimulate the exocytosis process of parietal cells, resulting in increased hydrogen chloride secretion in the stomach. In addition, less goblet cells were present in the villi of duodenum after the treatment of ML-SA1 (Figure 2B), further strengthening the notion that more protons were present in the gastric juice, since one of the functions of goblet cells is to secrete enough water and mucous to counteract the damage of low $\mathrm{pH}$ gastric juice to the intestine. To further confirm the observed phenomena, PAS staining was performed as shown in Figure $\mathbf{3 A}$ and $\mathbf{B}$. We can see from the graph that the amount of goblet cells was not significantly decreased in the duodenum sections of ML-SA1-treated mice. However, the stained sizes of goblet cells were drastically reduced, suggesting that majority of intracellular contents had been secreted. This is in accordance with the former observations that the acidity of gastric juice was increased.

\section{Application of ML-SA1 induced inflammation in the duodenum of mice.}

A common consequence of gastric juice acidification is increased inflammation of duodenum. To verify whether ML-SA1 induced gastric acid secretion, COX-2, an inflammation marker and inducer, was stained and quantified in the duodenum section of mice. As demonstrated in Figure 3C and D, a heavier staining of COX-2 were observed in the duodenum sections of ML-SA1 treated mice. In addition, COX-2 expression was mostly seen in the upmost region pointing towards the lumen of duodenum, which advised that the elevation of inflammation was restricted to the inner surface intestine.
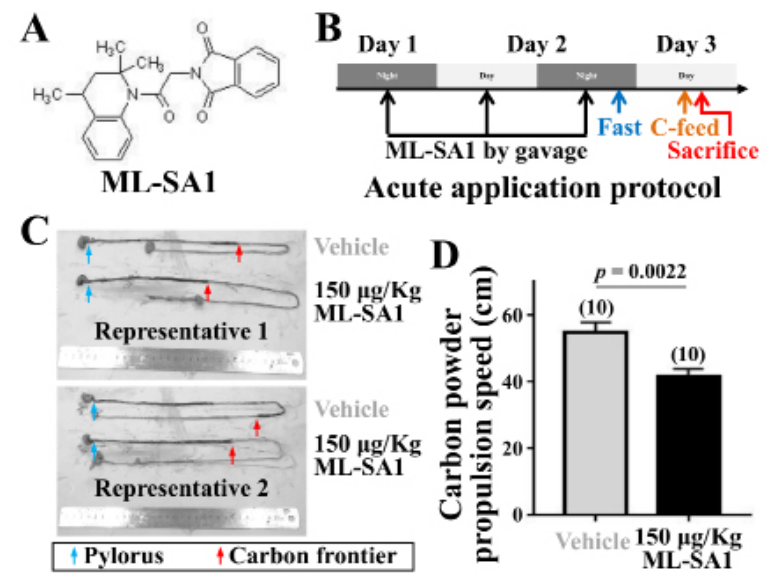

Figure 1. ML-SA1 treatment slowed Intestinal propulsion rate in mice. (A) The chemical structure of ML-SA1. (B) The detailed protocol of applying ML-SA1 (150 $\mu \mathrm{g} / \mathrm{kg}$, dissolved in DMSO as $10 \mathrm{mM}$ stock) to mice. (C) Representatives of the intestinal propulsion rate test. Blue arrow, the beginning of length measurement; Red arrow, the end of length measurement. (D) Summary of $(\mathrm{C})$. Numbers in the brackets indicated the number of mice used. Data were presented as mean \pm SEM. 
A

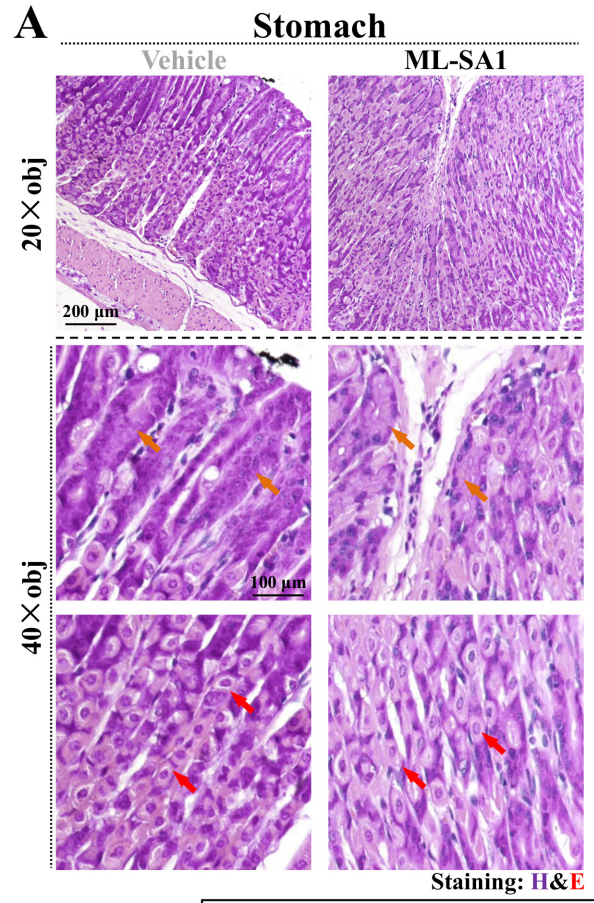

Surface mucous cells
B Duodenum

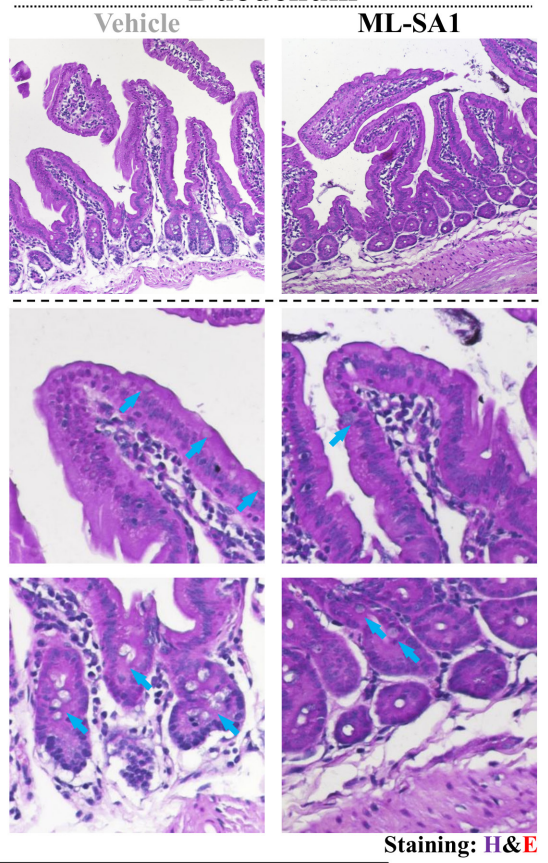

- Parietal cells

Goblet cells

Figure 2. ML-SA1 treatment altered the morphology of stomach and duodenum of mice. (A) The H\&E staining of stomach sections from the vehicle-treated or ML-SA1-treated mice. Orange arrow, surface mucous cells lining the up-edge of the pits; Red arrow, parietal cells lining the medium parts of the pits. Scale bar, $200 \mu \mathrm{m}$ for the $20 \times$ objective lens and $100 \mu \mathrm{m}$ for the $40 \times$ objective lens. (B) The H\&E staining of duodenum sections from the vehicle-treated or ML-SA1-treated mice. Blue arrow, goblet cells in the villi of intestine.

\section{Application of ML-SA1 increased the expression of TRPML1 in the GI tract of mice.}

ML-SA1 has been used as a specific TRPML1 channel agonist. Therefore, TRPML1 levels were assayed and monitored in both the stomach and duodenum sections of the mice. We can see that the expression of TRPML1 was specific, with TRPML1 mainly being observed in the parietal cells of stomach pits and in the columnar epithelial cells of duodenum villi (Figure 4A and B). The expression of TRPML1 was largely elevated in ML-SA1treated mice. It is also interesting to note that the pattern of TRPML1 expression was restricted as round circles inside cells, consistent with the previous observations that TRPML1 was mostly located in lysosomes and endosomes, but not on the plasma membrane of cells (10). This piece of evidence also pointed out the specificity of TRPML1 antibody since no staining was observed in other regions of the samples. Another meaningful finding was that TRPML1 was only observed in shrunken goblet cells in the duodenum but not in goblet cells possessing a round bubble, suggesting that the elevation of TRPML1 function would indeed increase the secretion of goblet cells in the intestine. Staining of the esophagus sections was used as a demonstration of efficacy of ML-SA1 on TRPML1 channel expression (Figure 4C).

\section{Application of ML-SA1 promoted TRPML1 expression and cytosolic calcium concentration in AGS cells.}

AGS cells, a stomach cell line, were used to demonstrate the aforementioned effect of ML-SA1 on the stomach. Various concentrations of ML-SA1 could induce a gradient rise of TRPML1 expression, as shown in Figure 5A. GCaMP5G, an exogenously expressed calcium sensor, was transfected into AGS cells. $20 \mu \mathrm{M}$ MLSA1 successfully triggered an elevation of intracellular calcium concentration in AGS cells. More importantly, micro-vesicles could be seen in Figure 5B, suggesting that ML-SA1 treatment might increase the exocytosis of AGS cells. Therefore, it would not be surprising to see an increased secretion of gastric juice in the stomach of mice after the application of ML-SA1.

\section{Discussions}

In this study, we demonstrated that gavage application of ML-SA1, a specific potent agonist of TRPML1 channel, could result in the enhancement of gastric acid secretion in the stomach of mice. As mentioned, a specific treatment of hypochlorhydria is lacking currently. Our research has highlighted a potential therapeutic agent that could be used for the treatment of hypochlorhydria. However, the following points are needed to be clarified first. We focused on the effect of ML-SA1 on GI tract in this study. 
As shown in Figure 4, the expression of TRPML1 channel was also elevated in the epithelia layer by the application of ML-SA1. The exact function of TRPML1 in the epithelia layer has not been established, and more investigations are needed to evaluate the safety of ML-SA1 in respect to other organs and tissues. In regards to the appropriate dosage of ML-SA1 to administer to hypochlorhydria patients, we hypothesize that $16.5 \mu \mathrm{g} / \mathrm{kg}$ would be a suitable dose based on the dosage given to mice. We observed that decreased $\mathrm{pH}$ in gastric juice caused an inflammation in the duodenum section of mice. This could be explained by the fact that normal mice were used instead of mice suffering from hypochlorhydria during the whole experiment. Therefore, efficacy and safety should be paid special attention to when ML-SA1 is introduced to mouse model of hypochlorhydria and subsequently human patients, since whether TRPML1 is still presented in the parietal cells during hypochlorhydria condition remains to be elucidated.

TRPML1 channel was believed to be a widely expressed protein in all tissues and cells. However, in our experiment, we found that this is not the case. In Figure 4, TRPML1 protein is only presented in parietal cells and columnar epithelial cells, but not in the surface mucous cells in the stomach or connective tissues in the duodenum. It could be possible that normal expression of TRPML1 is relatively low in many cell types, and therefore, IHC staining is not sensitive enough to detect it. However, it might be precisely regulated during physiological and pathological conditions, therefore application of ML-SA 1 could result in an increased TRPML1 expression. Moreover, the specificity of a certain cell type towards ML-SA1 could be a sign of importance of TRPML1 function in that particular cell type. For example, the fact that ML-SA1 induced increased TRPML1 expression in parietal cells and epithelium suggests that, normal function of TRPML1 might play a role in these cells. Taken into account the fact that the application of ML-SA1 led to a loss of intracellular content rather than a loss in the number of goblet cells, we speculate that TRPML1 could be a regulator of cellular exocytosis. As shown in Figure 5, ML-SA1 could indeed increase the expression level of TRPML1 channel, and upon its application increase cellular calcium concentration drastically.

Considerably, activation of TRPML1 via ML-SA1 initiated the formation of micro-vesicles in AGS cells, a stomach cell type, which further strengthened the notion that TRPML1 could regulate the exocytosis process of cells. This change happened relatively fast, since vesicles could be visualized only a couple of minutes upon the application of ML-SA1. Calcium has been closely correlated with cellular trafficking including endocytosis and
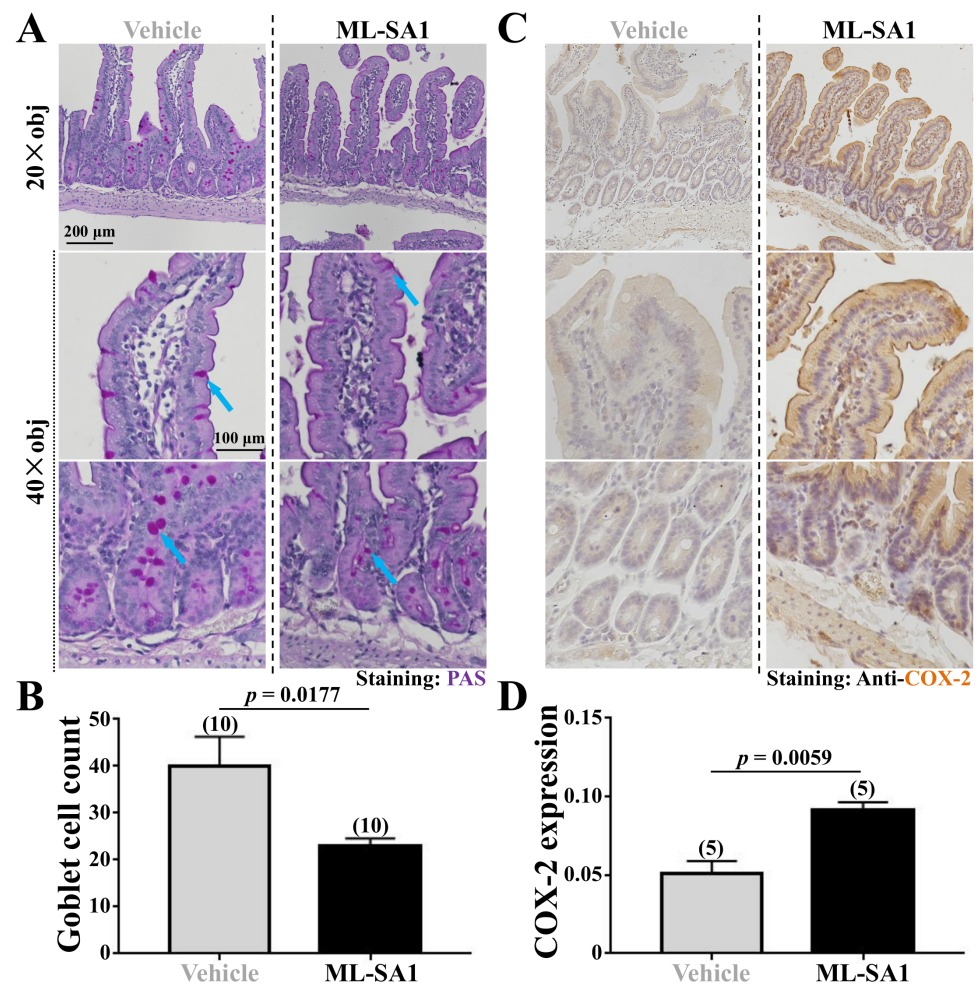

Figure 3. ML-SA1 treatment decreased the amount of goblet cells and induced inflammation in duodenum of mice. (A) PAS staining of duodenum sections from the vehicle-treated or ML-SA1-treated mice. Blue arrow, goblet cells that stained heavier. Scale bar, $200 \mu \mathrm{m}$ for the $20 \times$ objective lens and $100 \mu \mathrm{m}$ for the $40 \times$ objective lens. (B) Summary of (A). Numbers in the brackets indicated the number of sections from mice used. Data were presented as mean \pm SEM. (C) Immuno-histochemical staining of duodenum sections from the vehicle-treated or ML-SA1-treated mice. Scale bar, the same as (A). (D) Summary of (C). Numbers in the brackets indicated the number of sections from mice used. Data were presented as mean \pm SEM. 
A

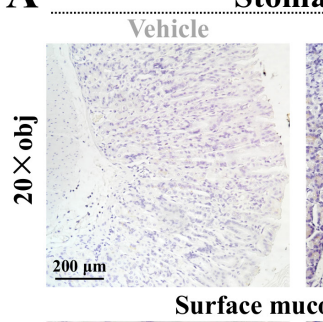

Stomach
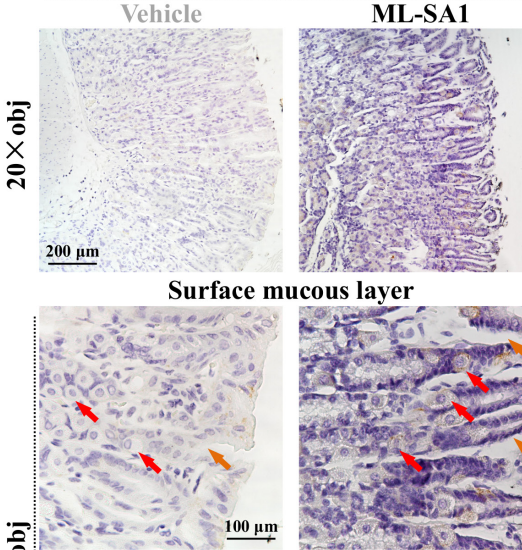

ucous layer

审.

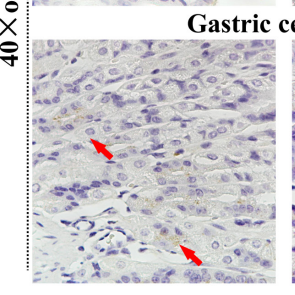

stric cell laye
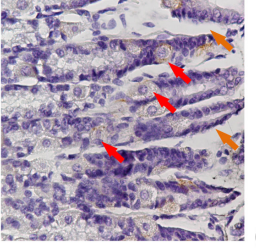

B

Duodenum

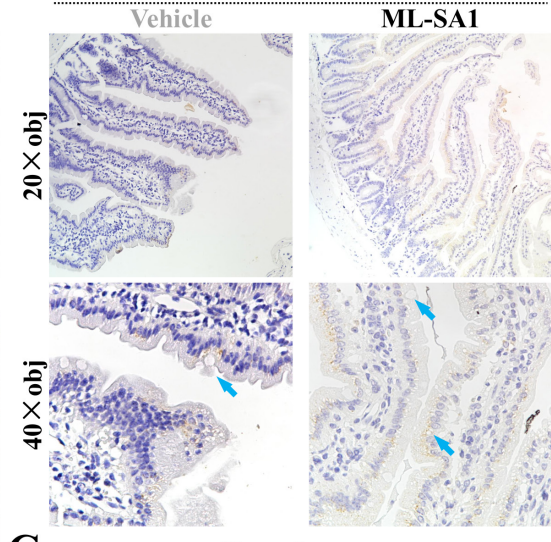

C

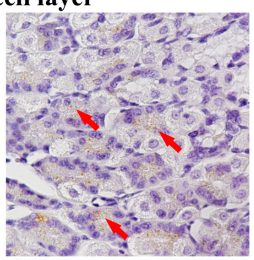

Staining: Anti-TRPML

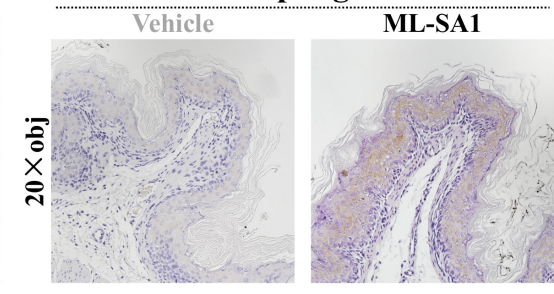

- Surface mucous cells

- Parietal cells

- Goblet cells

Figure 4. ML-SA1 treatment induced the expression of TRPML1 in vivo. (A) Immuno-histochemical staining of stomach sections from the vehicle-treated or ML-SA1-treated mice. Orange arrow, surface mucous cells lining the up-edge of the pits; Red arrow, parietal cells lining the medium parts of the pits. Scale bar, $200 \mu \mathrm{m}$ for the $20 \times$ objective lens and $100 \mu \mathrm{m}$ for the $40 \times$ objective lens. (B) Immunohistochemical staining of duodenum sections from the vehicle-treated or ML-SA1-treated mice. Blue arrow, goblet cells. Scale bar, the same as (A). (C) Immuno-histochemical staining of esophagus sections from the vehicle-treated or ML-SA1-treated mice. Scale bar, 200 $\mu \mathrm{m}$ for the $20 \times$ objective lens.

exocytosis (14). As a non-selective cation channel, TRPML1 is capable of transporting calcium from intracellular organelles into the cytosol (15). Therefore, it would not be too surprising to see an increased rate of exocytosis after the activation of TRPML1 by ML-SA1. While we only observed such phenomena in the gastric cell lines, it would also be interesting to test whether activation of TRPML1 could induce secretion in other cell types, especially in cells that intrinsically reside in endocrine tissues. Meanwhile, more investigations might be helpful to understand the detailed roles of TRPML1 during the secretion of cells.

Hypochlorhydria has been closely associated with, and has been suggested to be a hallmark symptom of gastric cancer. Currently, little is known about the causality between the two due to the lack of a specific activator of gastric juice production. Moreover, considering the recent discoveries that TRPML1 had been correlated with cancerous conditions (16-18), and that the activation of TRPML1 could be important for eliminating H. pylori (19), it would be plausible to further study the effect of ML-SA1 in various pathological conditions of stomach or even the GI tract. Doing so might allow us to further establish the correlations between TRPML1 and gastric cancer. In conclusion, our study provided a convincing attempt of applying ML-SA1 in animals, and pointed out a new possible research direction that TRPML1 channel could be a potential new therapeutic target in hypochlorhydria or even in the field of gastrointestinal cancer research.

\section{Conflict of interest}

The authors declare that they have no competing interests.

\section{Availability of data and material}

The datasets used and/or analyzed during the current study are available from the corresponding author on reasonable request.

\section{Consent for publication}

All authors have agreed to publish this article.

\section{Funding}

This work was supported by the National Natural Science Foundation of China (31400705) and Fundamental Research Funds for the Central Universities (CZY19027).

\section{References}

1. Fatima R, Aziz M. Achlorhydria. StatPearls. Treasure Island (FL)2019. 


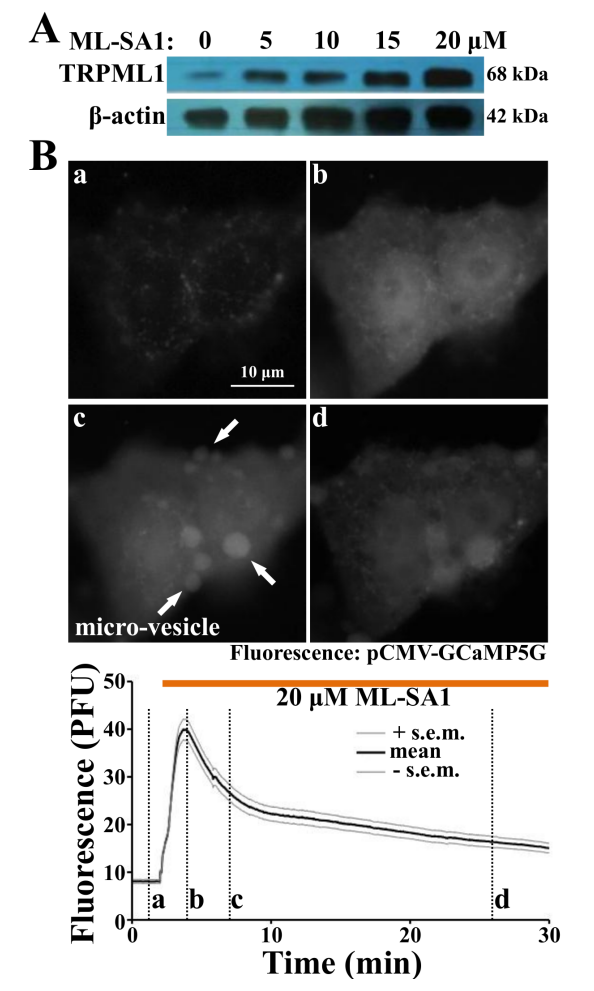

Figure 5. ML-SA1 activated TRPML1 in AGS cells. (A) Western blot of the TRPML1 expression in AGS cells after the application of various concentrations of ML-SA1. (B) The cytosolic calcium concentration change in calcium sensor transfected AGS cells after the treatment of $20 \mu \mathrm{M}$ ML-SA1. White arrow, micro-vesicles formed after the application of ML-SA1. Scale bar, $10 \mu \mathrm{m}$.

2. Belei OA, Heredea ER, Boeriu E, Marcovici TM, Cerbu S, Marginean $O$, et al. Verner-Morrison syndrome. Literature review. Rom J Morphol Embryol. 2017;58(2):371-6.

3. Giusti F, Marini F, Brandi ML. Multiple Endocrine Neoplasia Type 1. In: Adam MP, Ardinger HH, Pagon RA, Wallace SE, Bean LJH, Stephens K, et al., editors. GeneReviews $((\mathrm{R}))$. Seattle (WA)1993.

4. Lechner K, Fodinger M, Grisold W, Puspok A, Sillaber C [Vitamin B12 deficiency. New data on an old theme]. Wien Klin Wochenschr. 2005;117(17):579-91.

5. Sahoo N, Gu M, Zhang X, Raval N, Yang J, Bekier M, et al. Gastric acid secretion from parietal cells is mediated by a $\mathrm{Ca}(2+)$ efflux channel in the tubulovesicle. Dev Cell.
2017;41(3):262-73 e6.

6. Di Paola S, Scotto-Rosato A, Medina DL. TRPML1: The $\mathrm{Ca}((2+))$ retaker of the lysosome. Cell Calcium. 2018;69:112-21.

7. LaPlante JM, Falardeau J, Sun M, Kanazirska M, Brown EM, Slaugenhaupt SA, et al. Identification and characterization of the single channel function of human mucolipin-1 implicated in mucolipidosis type IV, a disorder affecting the lysosomal pathway. FEBS Lett. 2002;532(1-2):183-7.

8. Dong XP, Cheng X, Mills E, Delling M, Wang F, Kurz T, et al. The type IV mucolipidosis-associated protein TRPML1 is an endolysosomal iron release channel. Nature. 2008;455(7215):992-6.

9. Xu H, Delling M, Li L, Dong X, Clapham DE. Activating mutation in a mucolipin transient receptor potential channel leads to melanocyte loss in varitint-waddler mice. Proc Natl Acad Sci USA. 2007;104(46):18321-6.

10. Medina DL, Fraldi A, Bouche V, Annunziata F, Mansueto G, Spampanato C, et al. Transcriptional activation of lysosomal exocytosis promotes cellular clearance. Dev Cell. 2011;21(3):421-30.

11. Li X, Rydzewski N, Hider A, Zhang X, Yang J, Wang W, et al. A molecular mechanism to regulate lysosome motility for lysosome positioning and tubulation. Nat Cell Biol. 2016;18(4):404-17.

12. Clark R, Griffiths GM. Lytic granules, secretory lysosomes and disease. Curr Opin Immunol. 2003;15(5):516-21.

13. Shen D, Wang X, Li X, Zhang X, Yao Z, Dibble S, et al. Lipid storage disorders block lysosomal trafficking by inhibiting a TRP channel and lysosomal calcium release. Nat Commun. 2012;3:731.

14. Wu LG, Hamid E, Shin W, Chiang HC. Exocytosis and endocytosis: modes, functions, and coupling mechanisms. Annu Rev Physiol. 2014;76:301-31.

15. Gomez NM, Lu W, Lim JC, Kiselyov K, Campagno KE, Grishchuk $\mathrm{Y}$, et al. Robust lysosomal calcium signaling through channel TRPML1 is impaired by lysosomal lipid accumulation. FASEB J. 2018;32(2):782-94.

16. Grimm C, Bartel K, Vollmar AM, Biel M. Endolysosomal cation channels and cancer-a link with great potential. Pharmaceuticals. 2018;11(1).

17. Jung J, Cho KJ, Naji AK, Clemons KN, Wong CO, Villanueva $M$, et al. HRAS-driven cancer cells are vulnerable to TRPML1 inhibition. EMBO Rep. 2019;20(4).

18. Xu M, Almasi S, Yang Y, Yan C, Sterea AM, Rizvi Syeda AK, et al. The lysosomal TRPML1 channel regulates triple negative breast cancer development by promoting mTORC 1 and purinergic signaling pathways. Cell Calcium. 2019;79:80-8.

19. Capurro MI, Greenfield LK, Prashar A, Xia S, Abdullah M, Wong $\mathrm{H}$, et al. VacA generates a protective intracellular reservoir for Helicobacter pylori that is eliminated by activation of the lysosomal calcium channel TRPML1. Nat Microbiol. 2019;4(8):1411-23. 